\title{
Message size constraints on discourse planning in synchronous computer-mediated communication
}

\author{
CLAUDE G. ČECH and SHERRI L. CONDON \\ University of Southwesterm Louisiana, Lafayette, Louisiana
}

\begin{abstract}
Three groups of 20 dyads planned the MTV Music Video Awards Show over computers. The groups varied in whether they could send 4-line, 10-line, or 18-line messages, in part to examine whether increased planning efficiency in computer-mediated communication reflects communication strategies associated with constraints on message size. The results demonstrate that increased efficiency is not a function of such design features as the maximum message that may be sent. However, the subjects in the 4-line condition sent shorter messages to one another, were less likely to engage in a strategy of making multiple suggestions that required a single assent, and were more likely to differ in the relative proportion of their contributions to the discourse. The subjects in the 10-line condition had shorter maximum messages (and proportionately more disfluencies) than did those in the 18-line condition, despite the finding that the maximum messages of the latter would have also fit within 10 lines. Thus, the results also support a claim that size of the text window may result in different discourse management strategies and may influence an initial discourse-planning stage.
\end{abstract}

Computer-mediated communication (CMC) has become the focus of much interest (see, e.g., the recent volumes by Ess, 1996, and Herring, 1996). On first impression, it appears to be a very different form of communication than face-to-face conversation, especially when the many ways in which written discourse differs from spoken discourse are considered. Such an impression fails to take into account a number of considerations. One is that, despite some important differences in the two media (see, e.g., Ferreria \& Anes, 1994; see also Ko, 1996; Tannen, 1982), both written and spoken communicative discourse obviously share some communicative and speech act functions (although how much is shared is a matter for empirical investigation; see the review by Bordia, 1997). More to the point, although CMC is an increasingly important mode of discourse that is engaged in by an increasing number of people in their daily work and recreational activities, it in fact encompasses a variety of different systems with very different communicative features, ranging from UNIX TALK mode to e-mail to chat rooms and from IRC modes to multiuser domains (MUDs) and multiuser object-oriented domains (MOOs). Some of these are asynchronous (Kiesler, $\mathrm{Zu}$ brow, Moses, \& Geller, 1985) and, thus, differ with respect to important, possibly emergent features of spoken conversation, such as turn management and backchanneling (Clark \& Schaefer, 1989; Schegloff, 1968; Schegloff \&

This project was supported in part by Louisiana Educational Quality Support Fund Grant LEQSF (1996-98) - RD-A-38. We thank Michelle LeBlanc and Kelly Ryan-Biskup for their help with collecting and coding these data. S.L.C. is in the Department of English. Correspondence concerning this article should be addressed to C. G. Cech, Department of Psychology, University of Southwestern Louisiana, Lafayette, LA 70504 (e-mail: cech@usl.edu).
Sacks, 1973), not to mention persistence of message (email, for example, like written letters, can be read and reread at leisure while the respondent plans a reply). But some are synchronous, and thus might be expected to share more of the characteristics of spoken conversation. Although CMC systems are certainly worth investigating in their own right, the potential functional similarity of face-to-face interaction with interaction in some CMC systems provides an important avenue for new techniques with which to study discourse.

To take advantage of such new techniques, we need to be concerned with the very different computer interface design decisions implemented in studies that compare CMC and face-to-face communication (see, e.g., Baym, 1996; Condon \& Cech, 1996a, 1996b, 1998; Ko, 1996; Seu, Chang, Evens, Michael, \& Rovick, 1991). A major issue involves the question of which aspects of $\mathrm{CMC}$ are common to the medium itself and which emerge as a result of constraints imposed by features of the particular software being employed.

Although there are currently systems that enable synchronous communication (UNIX TALK mode, for example, or IRC), we created our own synchronous system in order to explore the consequences of various design features and, ultimately, to allow intervention in a conversation. Developing such a system requires a number of design decisions. Should interruptions be allowed (see Li, Seu, Evens, Michael, \& Rovick, 1992; Seu et al., 1991)? Should messages be sent as they are typed (truly on line) or after they have been composed (and thus, arguably, slightly off line)? Should split-screen techniques be employed that display the sender's and the receiver's messages simultaneously? Should we more thoroughly mimic auditory transience by erasing words a certain amount of time after they have been typed or received 
(a method that shares aspects of the moving window and RSVP methods)? Each of these decisions potentially affects communicative and compositional strategies and so may influence the resulting conversation. For example, use of split-screen, immediate, bidirectional communication may allow opportunities, not present in forcedturn communication, to study claims that turns are emergent phenomena (see, e.g., Clark, 1994) rather than necessary means of structuring the conversation (see Anderson, Beard, \& Walther, 1998). At the same time, split-screen techniques potentially alter the involvement of working memory by allowing review of one's own message while reading a reply. As this example illustrates, specific design decisions may alter the results obtained with $\mathrm{CMC}$ in profound ways.

In this study, we explore the greater efficiency of computer talk, as reflected in two possibly related characteristics of CMC: the commonly reported finding that shorter and fewer utterances accomplish conversational goals and our own previously reported finding that computer talk reflects stronger adherence to a discourse-planning schema. At issue are the robustness of these findings across different software designs and conversational goals and whether the findings reflect the same underlying mechanism. We also present evidence that software design may influence the planning strategies and relative verbosity of discourse partners.

\section{CMC Software}

We developed software that approximated some features of face-to-face conversation (Condon \& Čech, 1996b). The program allowed the sending of multiple messages, subject to a constraint of a maximum of slightly over three lines per message. Pressing the Enter key after composing a message sent it to the partner and erased any previous message on the partner's screen. To partially mimic the transient character of spoken communication, the sender's message disappeared from her screen on transmission, and messages received similarly disappeared when subjects started typing their replies. Elementary correction (backspacing over the current line) was enabled in order to examine disfluencies and shifts in communicative strategy.

The software allowed people to interrupt one another, with similar consequences: An interruption erased the message on the recipient's terminal, although that message stayed in the buffer, and any further typing done by the interrupted partner was appended to the message before being sent on (see Condon \& Čech, 1996a, 1996b, for further details).

\section{The Schematic Discourse Model}

Condon and Čech (1996b) formulated a high-level schema for problem-solving discourses in which a goal is established, input relevant to satisfying that goal is proposed, evaluation of the input occurs, and, on the basis of the evaluation, the proposal is adopted (consensus), and the next goal takes precedence. This schema structures the discourse, because schema functions are associated with utterance functions in routine sequences. The routine utterance function for suggesting a goal is orientation, whereas the routine utterance function for input should be making a suggestion. The routine function for evaluation and consensus, of course, is agreement.

Condon and Cech (1996b) argue that, when utterance functions are linked in routines, they resemble adjacency pairs (Levinson, 1983; Sacks, 1973) that consist of a first pair-part that creates a slot for and constrains a second pair-part. For example, a question calls for a response as the second pair-part and a request for a compliance. Thus, the default response to a formulated goal is an input (suggestion), and to the input is a positive evaluation (agreement). Defaults in discourse schemas and routines carry the benefit of reducing the amount of linguistic encoding that is needed to make decisions: Defaults, being expected, can be encoded in less linguistic form than can dispreferred (marked) functions (Levinson, 1983) and, in the extreme, need be encoded in no linguistic form at all. On the basis of these expectations, the simple routine that appears in streamlined problem-solving conversation may, thus, be characterized in terms of a recursive series involving orientation, suggestion, and agreement (see, also, Hiltz, Johnson, \& Turoff, 1986).

We analyzed 16 computer-mediated and 16 face-toface conversations for conformity with the schema. The discourses involved two variants of planning a simple itinerary (a visit or a weekend getaway) or a simple social event (a picnic or a barbecue). The conversations were separated into utterance-level units (single clauses with complements and adjuncts; see Condon \& Čech, 1996b, for details) and were characterized according to an utterance-coding system (after Condon, Cooper, \& Grotevant, 1984) that assigned a value for each of three broad categories of utterance function: Moves, Responses, and Other. Moves included the functions Suggests Action, Requests Information, Requests Validation, Requests Action, and Elaborates/Repeats; Responses included the functions Agrees with Suggestion, Disagrees, Complies with Request, and Acknowledges Only; and Other included the use of Discourse Markers, Metalanguage, Personal Information, Joking/Exaggeration, as well as the function Orients Suggestion. When none of the functions in a given category was appropriate, we assigned it a No Clear code. (See Condon \& Čech, 1992, for further details of the coding system. A revised version of this coding manual is available on line from the Discourse Re-

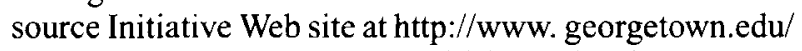
luperfoy/Discourse-Treebank/dri-home.html)

Despite striking differences in the number of utterances in the two modalities ( 259 vs. 57 utterances, on the average, for face-to-face and CMCs, respectively; see also Lea, O'Shea, Fung, \& Spears, 1992; Sproull \& Kiesler, 1991), the relative proportions of utterance functions in each were remarkably uniform over the four problems. We found very similar ratios across modality for three major predicted sequences in the discourse routines: 
agreements following suggestions, compliances following requests, and suggestions following orientations.

Nevertheless, there were important differences. Our $\mathrm{CMC}$ and face-to-face dyads had different proportions for most of the coded functions, and the CMC dyads appeared to engage in more efficient communication, as evidenced by a much higher proportion of suggestions ( $29 \%$, as opposed to $18 \%$ in the face-to-face condition). Requests and orientations were also more common in CMCs (although face-to-face communication and CMC failed to differ in the virtually nonexistent dispreferred utterance function of Disagreement). Such results (including more utterances combining functions in CMCs) have led us to claim that CMC is more efficient in the system we have implemented, a finding we have termed compression (Condon \& Čech, 1996a). From an attentional perspective, compression makes sense: Typing at a keyboard for most people is more time consuming than is speaking (see, e.g., Siegel, Dubrovsky, Kiesler, \& McGuire, 1986) and forces the partner to maintain continued topic focus while a response is being prepared. Emergent routines that minimize attentional demands are thus likely, selecting for relatively small and rapid messages (see Colomb \& Simutis, 1996; Hiltz et al., 1986; Weisband, 1992).

But to what extent is compression related to sending short messages? Short messages may have increased efficiency by stripping away extraneous linguistic material (or unnecessary elaborations) not fitting the plan categories. That possibility, in turn, suggests that increased efficiency may have reflected design features of the software rather than the medium itself. Given the message size constraints and the instructions to people to type short messages that fit within three lines, we may have biased subjects to compose short turns that could be completed in a single exchange of messages. Such a bias may have prevented people from transmitting a preferred message size. Is there an optimal message size that would have changed the amount of conformance to the discourse schema model? To investigate these issues and broaden the generality of the earlier findings, the current study manipulated several design features and presented the subjects with a more complex planning task.

\section{METHOD}

The subjects planned the MTV Music Video Awards Show over computers. Pairs of subjects were randomly assigned to one of three conditions that represented the maximum message or broadcast (4-line, 10-line, or 18-line message) that could be composed.

\section{Materials and Software}

In part because of the virtually nonexistent levels of Disagreements in the earlier tasks, we selected a new task that we thought would be more likely to elicit some strong opinions and, thus, potential for disagreement: planning the MTV Music Video Awards. In the earlier tasks, the subjects filled out a plan sheet in order to ensure a minimum number of decisions. The plan sheet in the itinerary conditions, for example, indicated activities for various times of the day. We eliminated the plan sheet for the MTV problem, for several reasons. One involved avoiding the time that would be spent negotiating about who would write what down when on the plan sheet (not to mention the periods of conversational inactivity while the writing was taking place). Another involved avoiding use of a consultable record of the decisions already made (reducing memory load). A third concerned the possibility that the plan sheet structured the conversation too strongly in terms of telling subjects what their subgoals ought to be. Although we could not totally avoid the structuring problem, we did try to be relatively nonspecific about the numbers of decisions (e.g., how many categories or nominees) people could choose to make. Thus, the following guidelines remained at the bottom of the screen during the task.

We want you two to plan the MTV Music Video Awards! So, you two will need to pick a number of CATEGORIES, decide who the NOMINEES ought to be, decide what CLIPS to show of their videos, decide who the WINNER should be, decide who ought to be PRESENTING the nominees and the awards for each category, etc. Don't forget that there is an OPENING ACT for the show, and that several BANDS perform between awards: You get to choose who they ought to be, as well! In short, plan what you think will be music video awards show people will be talking about for days and weeks after. Enjoy!

The MTV problem also represented a significantly more complex problem than did the earlier tasks, in order to allow the assessment of robustness of the discourse model over problem complexity.

There were also several changes in the software. The most significant of these concerned text window size. In the current program, the subjects could type and receive messages within a text window consisting of 4,10 , or 18 lines (of 80 characters each). The window, framed at top and bottom by a border, appeared in the top half of the screen. A warning signal and message appeared ("BUFFER ALMOST FULL: You're on the last line, finish it, and send this bit!'") when the composer's text reached the last line of the window.

Varying the text window allows examining whether compression is an artifact of text window size. It also provides additional benefits. As indicated earlier, CMC often imposes attentional demands that differ from those found in face-to-face communication (see Lea et al., 1992; Spears \& Lea, 1994). We think these demands may be partly responsible for compression. But systematically varying message size arguably imposes yet further demands on attentional resources. Thus, the 18-line condition has the benefit of enabling a rich and long message to be sent, but at the severe cost of a relatively long wait and the need for maintained topic focus by the recipient. Conversely, the 4-line condition limits the typing that needs to be done and, in theory, allows fairly rapid responding (although one could argue that trying to plan a message within 4 lines frontloads attentional demands). If a 4-line window is insufficient for efficient communication, we might expect to see longer messages in the 10-line and 18-line windows. Conversely, the relatively long time it takes to type more than 10 lines (coupled with a long wait on the recipient's part) might lead us to predict that an upper limit on length of messages would render the latter two conditions equivalent. Thus, varying the window size from 4 to 18 lines also enables us to determine (1) whether there is some preferred message length that subjects settle on, independently of window size (at least, in the large-window conditions), given the choice, and (2) whether variations in message size correspond with variations in adherence to the discourse schema, as would be expected if compression results from or strongly correlates with choices that affect message size.

There were also other, less dramatic changes in the software. A one-line message window below the text window displayed blinking status messages, including the last-line warning message mentioned earlier, and messages that one's partner had started typing or was adding to a previous transmission. These status messages appeared in different colors in order to make them distinctive. We also enabled backspacing over lines rather than just within a line and provided a digital clock in the top right corner of the screen that up- 
dated whenever a message was sent, as previous subjects sometimes had to be cued to finish within the allowed time. Finally, a followup message was added to and caused scrolling (rather than erasing) of the previous message on the recipient's screen. However, the subjects could not view any part of the (earlier) message that had scrolled off their text window. As before, a message composed by a subject was erased and replaced by the incoming message of the partner.

\section{Procedure}

The subjects were informed of the task and placed on computers in separate rooms. The computers provided instructions about how to communicate. Specifically, the subjects composed messages and used the Enter key to send them to one another. When they had finished the task, they pressed the Escape key. To ensure a minimum number of exchanges, the Escape key was enabled only after at least three exchanges.

The subjects could send as many messages in a row as they desired, in order to overcome the limitations of a window that did not provide sufficient room for the desired communication. All keystrokes (including backspaces) and the times at which a message was initiated and sent were recorded. A maximum of $2 \mathrm{~h}$ was allowed for the task, although not all teams required the full time.

\section{Subjects}

One hundred twenty students at the University of Southwestern Louisiana participated in exchange for bonus credit in introductory psychology. These students were randomly assigned to one of the three text window conditions.

\section{RESULTS AND DISCUSSION}

An overview of the three conditions appears in the first section below. Subsequent sections restrict analyses to just the extreme (4-line and 18-line) conditions, in order to maximize contrast.

\section{Turns and Messages}

We will start with the results concerning message sizes and numbers of transmissions. As a first pass through the data, we analyzed the data, using both analyses of variance (ANOVAs), where appropriate, and contrast analyses. With respect to the latter, we employed a linear contrast and a contrast in which identical 10-line and 18-line conditions were opposed to the 4-line condition, specifically in order to investigate the issue of optimum message size. In several cases, the variances differed enough that we used other statistics (such as Welch's $W$, which does not assume equal variances) rather than an ANOVA or analyzed reduced data sets.

We first looked at whether there were different amounts of information conveyed in the three conditions. There are several ways of asking this question, but the first that we examined concerned file size: Did people in the 4-line condition have smaller conversations? Our measure of file size essentially counted the number of characters transmitted (including spaces) divided by 80 , in order to give a measure of file lines. There was no significant difference in file size among the three groups $(F<1)$ for the ANOVA and the contrasts. On average, our CMC files contained 55.75 lines. This is almost twice the number of lines ob- tained in the earlier CMC condition (24.61 lines), which supports the claim that the MTV task was more complex.

We next looked at average number of lines and words sent per broadcast, in order to see whether message size increased with window size. Although the ANOVA in each case was nonsignificant, the variances were generally too different (much higher variance in the 10-line and 18-line conditions) to allow much confidence in the analysis. Using the Welch test, we found evidence that messages were somewhat shorter in the 4-line condition, in which people sent an average of 1.103 lines per transmission (half a line less material than in the other two conditions; $W(2,32)=3.26, p=.052)$, and 16.8 words (7 words less than in the other conditions; $W(2,31)=3.42$, $p<.05)$. ANOVAs on just the 10 -line and 18 -line conditions verified their essential equality (both $F_{\mathrm{s}}<1$ ). These message sizes represent a slight increase over previous results obtained with the itinerary and social event problems (0.796 lines and 10.26 words, for the CMC condition).

We also looked at the total number of messages or broadcasts in order to see whether the 4-line window subjects sent more messages in order to make up for the reduced window size. As might be predicted from the short average sizes of messages presented above, they did not $[W(2,33)=1.7, p>.19]$. In fact, the number of broadcasts by the 4-line group (50.9) fell in between the numbers of broadcasts by the 10-line (63.0) and the 18-line (41.0) groups.

The data on number of messages broadcast suggest that there ought not to be much difference among groups if we look, instead, at total number of turns (we define turn boundaries at transitions to the partner). Consistent with this expectation, the subjects generally took the same number of turns in all conditions $[W(2,33)=1.63, p>$ $.21]$. They did occasionally send several messages in a row, however, as there were fewer turns, on average, than broadcasts ( 43.5 vs. 51.6). People generally had less to say per turn in the 4-line condition (18.6 words) than in the other two conditions [ 29.5 words; $W(2,29)=3.77, p<.05]$.

We next evaluated the maximum message that was sent in a given conversation. Table 1 illustrates the maximum messages (in terms of lines) for each subject in the three conditions. As is evident from these data, there was a robust effect that was due partly to the limitations of a 4-line screen. But the data for the 10-line and 18-line conditions also suggest more than a ceiling effect. Because of the very high variance in these two conditions, we analyzed these data with a nonparametric test, the Mann-Whitney test. The results supported a preliminary determination of larger messages in the 18-line condition $[z=1.891, p<.06$ (two-tailed)]. Thus, we obtained some preliminary evidence against an optimal sized message that would fit within a 10 -line window, although most of the subjects in this condition had maximum messages that were well under 10 lines.

The data in Table 1 illustrate a further interesting result: Within the 18-line condition, only one subject ever had a maximum message of over 10 lines (as did a sub- 
Table 1

Maximum Message (in Lines) Sent by First (P1) and Second (P2) Partner in the Three Text-Window Conditions

\begin{tabular}{|c|c|c|c|c|c|c|}
\hline \multirow[b]{2}{*}{ Dyad } & \multicolumn{2}{|c|}{ 4-Line Window } & \multicolumn{2}{|c|}{ 10-Line Window } & \multicolumn{2}{|c|}{ 18-Line Window } \\
\hline & P1 & $\mathrm{P} 2$ & P1 & $\mathrm{P} 2$ & P1 & $\mathbf{P} 2$ \\
\hline 1 & 2.16 & 1.95 & 1.69 & 2.25 & 1.21 & 2.53 \\
\hline 2 & 1.83 & 2.13 & 3.55 & 1.25 & 4.57 & 6.44 \\
\hline 3 & 2.10 & 1.45 & 2.80 & 1.63 & 3.40 & 4.70 \\
\hline 4 & 2.70 & 3.83 & 4.65 & 4.14 & 2.66 & 7.35 \\
\hline 5 & 2.94 & 3.19 & 3.59 & 9.11 & 7.26 & 8.30 \\
\hline 6 & 2.47 & 2.75 & 3.89 & 4.99 & 8.26 & 5.09 \\
\hline 7 & 1.81 & 3.35 & 3.96 & 2.20 & 6.68 & 9.31 \\
\hline 8 & 3.63 & 2.40 & 4.16 & 2.96 & 2.01 & 1.00 \\
\hline 9 & 3.64 & 2.96 & 3.41 & 6.35 & 3.35 & 2.83 \\
\hline 10 & 2.25 & 3.01 & 5.64 & 4.24 & 3.60 & 1.69 \\
\hline 11 & 4.40 & 3.97 & 4.13 & 7.32 & 6.95 & 7.03 \\
\hline 12 & 1.69 & 1.76 & 3.51 & 1,00 & 4.54 & 1.25 \\
\hline 13 & 3.20 & 2.69 & 4.75 & 3.40 & 3.38 & 2.76 \\
\hline 14 & 1.39 & 2.11 & 3.78 & 1.66 & 3.20 & 5.05 \\
\hline 15 & 2.20 & 1.89 & 3.80 & 3.67 & 9.44 & 4.71 \\
\hline 16 & 2.78 & 3.01 & 13.15 & 9.00 & 5.19 & 6.96 \\
\hline 17 & 2.96 & 3.95 & 5.69 & 4.06 & 2.58 & 4.25 \\
\hline 18 & 3.06 & 3.97 & 1.56 & 2.20 & 8.99 & 4.51 \\
\hline 19 & 3.41 & 2.67 & 1.35 & 2.42 & 12.19 & 5.21 \\
\hline 20 & 1.96 & 2.56 & 4.66 & 3.66 & 4.16 & 6.15 \\
\hline Mean & 2.63 & 2.78 & 4.19 & 3.88 & 5.18 & 4.86 \\
\hline
\end{tabular}

ject in the 10-line condition). Thus, the general trend toward longer maximum messages in the 18-line condition does not appear to be due to a need to exceed a limit of 10 lines. Rather, these data strongly suggest that the increase is to be accounted for in a message-planning stage: Providing a 10-line text window apparently establishes some a priori constraints on how much information people think they can include (at least in the early stages of a message), even though producing smaller messages will prove unnecessary because virtually all messages will fit within 10 lines.

If this analysis of the longer maximum messages in the 18 -line condition is correct, the subjects in the 10 line condition should have had greater difficulty composing messages to fit planning constraints. If, as in spoken language (see, e.g., Clark, 1996), people started producing messages before they were fully planned or compiled, we ought to see evidence of greater disfluency in the 10-line than in the 18-line condition. To assess disfluency, we looked at the correlations of proportion of backspacing with maximum messages (in lines), following the logic that subjects in the 10-line condition composing long messages require more attentional resources to meet planning constraints. Supporting a disfluency claim, there was a strong correlation for the 10 -line subjects $[r=.63, t(38)=5.03, p<.01]$ but no correlation $(r=.05)$ for the 18-line subjects. Given that the 4-line condition forces much smaller messages, we did not expect a similar correlation; the smaller size of the message would seem to impose much more severe constraints on planning prior to starting to type. Indeed, the correlation in this latter condition was marginal, at best, and in the wrong direction $[r=-.26, t(38)=-1.64]$. Thus, the analysis of disfluencies lends support to the claims of a planning stage effect.

A final result in this initial overview concerns the positive correlation between the average message sizes partners sent one another $[r=.52, .76$, and .69 in the three respective conditions, $\min t(18)>2.58, p<.01]$. These correlations did not differ significantly.

\section{Compression}

We asked earlier whether the higher rates of conformity to the discourse schema in CMC might have been due to the use of a small message size or to instructions to keep messages short. The latter possibility seems unlikely, given the average short messages we obtained. Nevertheless, in order to address this question, we separated the messages of the 4-line and 18-line discourses into utterance units and coded the units into utterance categories. The two conditions were comparable in terms of the average number of utterances (62 and 60, respectively). Despite the fact that our file sizes were larger in the current experiment, these figures were equivalent to the number of utterances (57) that subjects in our earlier CMC task exhibited.

Given that the 4-line and 18-line conditions represent the extremes in terms of message size constraints, a claim that message size moderates compression predicts greater compression in the 4-line condition and, thus, greater proportions of utterance functions that meet the discourse schema. Although we have refined our code categories considerably since the earlier papers, we here report on the categories that are comparable to those used earlier (Condon \& Čech, 1996a, 1996b), so that interested readers may compare the results. The relevant data appear in Figure 1.

As is strikingly obvious from this figure, the 4-line and 18-line conditions were much alike on all code categories (which is true, as well, for the new code categories not discussed in this article). In separate ANOVAs on the three broad categories (Moves, Responses, Other), the only effect significant at conventional levels involved the lowered proportions of Response functions $(5.7 \% \mathrm{vs}$. $7.1 \%)$ in the 18 -line window $\left[F(1,38)=6.2, M S_{\mathrm{e}}=\right.$ $0.0012, p<.02]$. Although this finding is consistent with a claim that compression moderates with window and/or message size, the lack of any such finding in the proportions of Moves $(F<1)$ strongly suggests an alternative explanation: People in the 18-line condition were more likely to use a planning strategy of encoding several suggestions per turn, requiring a single assent (and thus proportionately fewer explicit Response functions; see, also, the discussion of density below).

The CMC results from the earlier experiment (Condon \& Cech, 1996b) also appear in Figure 1. The current results were very much like those found earlier. To the extent that Suggests Action serves as a rough guide to the amount of problem solving, the subjects in these different experiments made approximately the same num- 


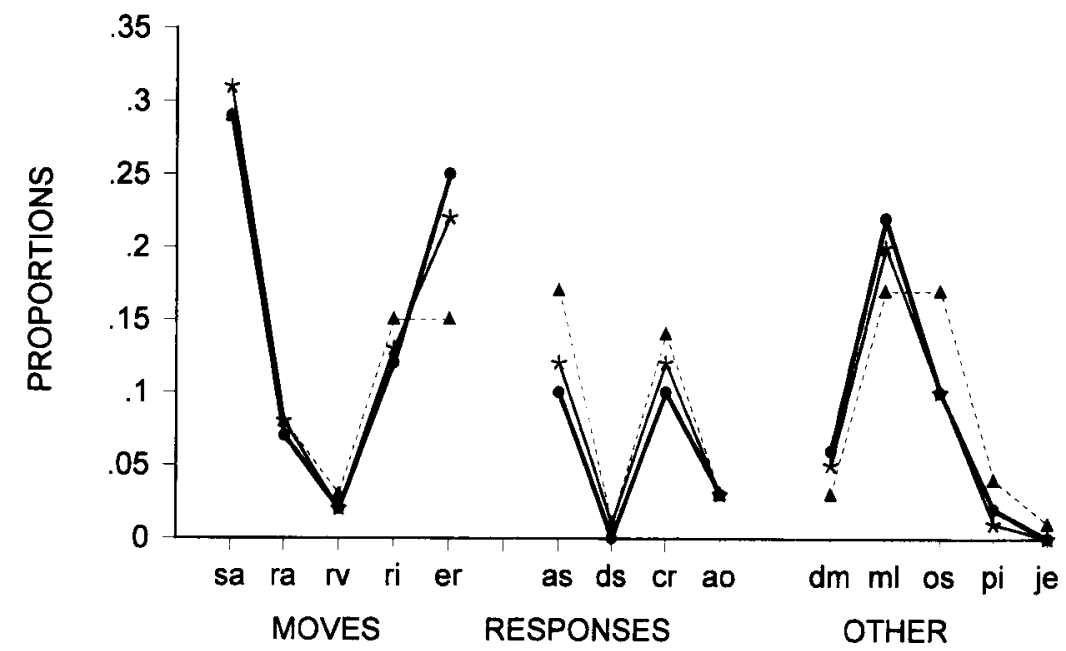

Figure 1. Proportions of Moves, Responses, and Other utterance functions in the 4-line (asterisks) and 18-line (circles) conditions and in Condon and Čech's (1996a) 3-line condition (triangles). Move functions include Suggests Action (sa), Requests Action (ra), Requests Validation (rv), Requests Information (ri), and Elaborates/Repeats (er). Response functions include Agrees and Disagrees with Suggestions (as and ds), Complies with Request (cr), and Acknowledges Only (ao). Other functions include Discourse Marker (dm), Metalanguage (ml), Orients Suggestion (os), Personal Information (pi), and Joking/ Exaggeration (je).

hers of decisions. With a linear regression analysis, we assessed the similarity of results across experiments, using the earlier CMC results to predict the proportions of code categories in the 4-line and 18-line conditions. This analysis found a reasonably good fit $\left(R^{2}=.817\right.$, $p<.001$ ) that diminished when the earlier face-to-face proportions were used as the predictor variable $\left(R^{2}=\right.$ $.511, p<.001)$. These results support our earlier observation that the patterning of results in CMC differs substantially from that found with face-to-face planning.

Nevertheless, Figure 1 shows that there were indeed some differences from the earlier CMC results, including the heightened number of elaborations in the current conditions and the lower numbers of orientations and agreements. The latter is consistent with long-message strategies in which a single orientation can be followed by a number of suggestions, all of which are adopted through a single agreement. The increase in elaborations can be attributed to several tendencies. One consisted of long sequences of elaborations that were used to establish referents and to recall names. Another involved using elaboration sequences to support suggestions and agreements. Given the longer turns in the current studies, we suspect that this latter tendency may reflect a function similar to backchanneling (Clark \& Shaeffer, 1989; Schegloff, 1982), whereby one subject signals continued attention to the discourse of another subject.

As before, there were virtually no disagreements. The data demonstrate a very strong cooperative bias, in contrast to the disagreements and flame wars often found in Usenet groups (see Baym, 1996; see also Lea et al., 1992). Of course, cooperativity is the default in our model.

\section{Other Window-Size Findings}

We obtained two other findings that appear to moderate with window size. One concerns the above-mentioned tendency for subjects in the large-window conditions to adopt a strategy of planning that involves a small number of long turns, and the other concerns the tendency for one subject in the small-window condition to send longer messages than the other. We refer to these tendencies, respectively, as density and dominance.

Figure 2 illustrates density in the two conditions. It plots mean words per turn against number of turns. At one extreme are several conversations that involve a large number of turns involving exchanges of relatively few words. At the other is a conversation that finishes in very few but very large turns. Indeed, although a power or exponential function could be fit to these data, the latter conversation seems to involve a qualitatively different approach on the part of the subjects. The figure illustrates the extremely high variability in these conversations.

How well does a linear function fit these data, if we remove the extreme high-density outlier? In fact, a linear function does a very poor job of explaining the results for the four-line condition $(r=-.14)$. However, there is a significant inverse correlation between number of turns and average turn size in the other condition, once the outlier has been removed $[r=-.67, t(17)<-3.73, p<$ .002]. Although the subjects in the four-line condition had the option of creating a long turn by sending several messages in a row, they generally did not employ this strategy.

We operationalized density as number of words per turn divided by the number of turns. The results indicated that average density increased in the 18-line condition 


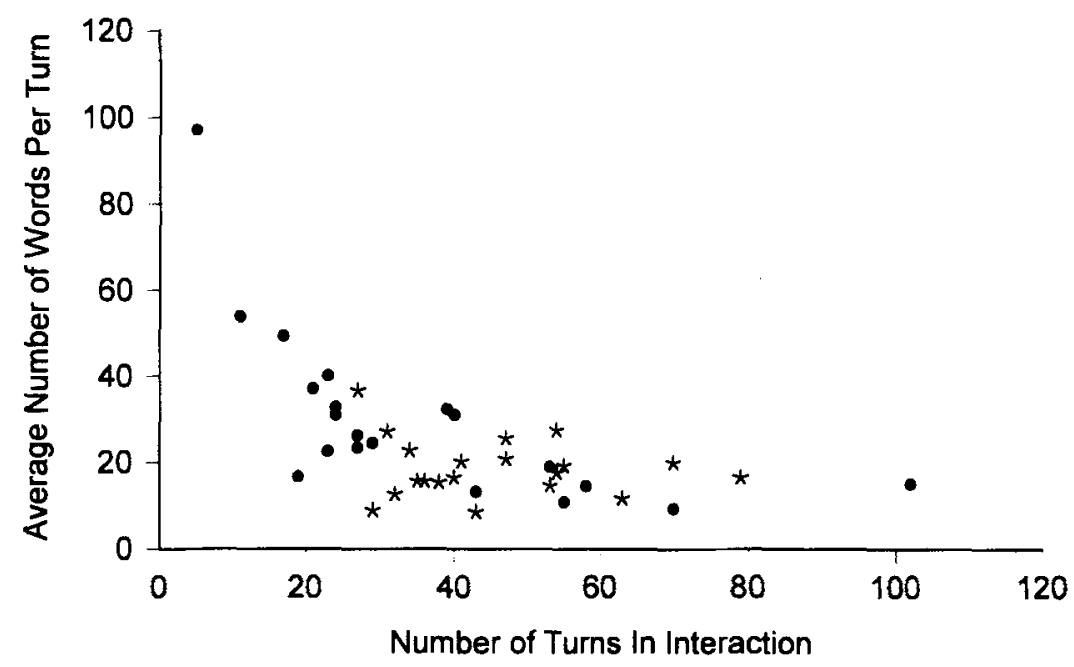

Figure 2. Scatterplot of average turn size and number of turns for the 4-line (asterisks) and 18-line (circles) conditions.

[from 0.45 to 2.04 ; with the outlier removed in the 18 line condition, average density is $1.13 ; W(1,20)=6.29$, $p<.025$, for the latter condition].

Why is density important? We hypothesized that, as density increases, there is a shift in management strategies from one in which partners cooperate in micromanaging every decision to one in which they adopt a strategy of broadcasting a number of potential decisions at once (a hierarchical planning strategy that involves formulating a number of suggestions and orientations in a single turn; see the discussion of parallel strategies and long turns in Condon \& Cech, 1998). To evaluate this claim, we correlated the average number of suggestions and requests per turn with density in the two conditions. The correlation was quite impressive for suggestions $(r=.96)$, although there was no correlation for requests $(r=.05)$. When we looked at the 4-line and 18-line conditions separately, the correlation of density with number of suggestions per turn remained high $(r=.70$ and .98 , respectively), even when we removed the one extremedensity conversation in the 18-line condition $(r=.88)$. In fact, when we reexamined the correlations, combining the two window conditions but removing this anomalous pair, we found high correlations of density not only with suggestions $(r=.88)$ but also with requests $(r=.78)$. There was also a moderate but significant correlation of density with the average number of compliances with requests $[r=.44 ; t(37)=2.98, p<.005]$ and a correlation with agreements per turn $(r=.26)$ that approached a conventional level of significance $[t(37)=1.64, p<.06]$. Because a hierarchical strategy should increase the ratio of suggestions and requests to their preferred responses (compliances and agreements), the correlations ought to be stronger for requests and suggestions.

We also examined the extent to which one partner seemed to dominate the conversation. Hiltz et al. (1986) claimed that $\mathrm{CMC}$ is a more egalitarian medium, although Spears and Lea (1994) suggested that this result may reflect demand characteristics of CMC. We were interested in the extent to which one measure relevant to this claim, relative verbosity, was (like density) a negotiated discourse management strategy responsive to design features of the software being used.

We measured dominance in several different ways. One measure involved a simple count of the proportion of turns on which one partner had more to say. The other measure tried to take into account whether a given subject engaged in only slightly longer or much longer turns, by employing the ratio of the more verbose to the less verbose partner (defined over average number of words per turn) multiplied by the proportion of turns on which the more verbose partner had a longer message. The expected value for egalitarian dyads on both measures is .5. The 4-line condition had a slightly higher level of dominance than did the 18 -line condition $[0.681$ vs. .588 for proportion of turns, and $1.114 \mathrm{vs.} 0.870$ for the modified ratio; $F(1,38)=6.95, M S_{\mathrm{e}}=0.0125, p<.02 ; F(1,38)=3.98$, $\left.M S_{\mathrm{e}}=0.1487, p<.054\right]$.

We also did some preliminary analyses in order to evaluate whether dominance involves one partner controlling the conversation by making more requests or suggestions. These correlated dominance with the difference in proportion of certain code categories (suggestions, requests, elaborations, and orientations) between the more verbose and the less verbose partner. The highest correlations occurred with the proportion of turns measure. Somewhat to our surprise, the correlations with suggestions and requests were nonsignificant $(r=-.02$ and .19 , respectively), although there was a reliable correlation with elaborations $[r=.49 ; t(38)=3.47, p<.005]$ and some suggestion of a correlation with orientations $[r=.26 ; t(38)=1.66, p<.06]$. The correlation with 
elaborations remained significant when we examined the 4-line and 18-line conditions separately $(r=.50$ and .59 , respectively).

Thus, the results we obtained with dominance suggest that it, like density, is in part an emergent feature of software design: Dominance and density are not equally likely in the different window conditions, despite the fact that each condition in principle allowed the concatenation of long turns composed of a series of broadcasts.

\section{CONCLUSIONS}

The results support the claim of Condon and Cech (1996a, 1996b) that dyadic planning in synchronous $\mathrm{CMC}$ exhibits compression - an increase in task-related talk that represents a heightened conformity to the schematic discourse-processing model presented earlier. Others have commented on a similar finding (see, e.g., Hiltz et al., 1986), but our data now eliminate several possible explanations for that finding. We have been able to show that compression need not be an artifact of requiring people to transmit small messages. Similarly, the change in average message size from the 4-line condition to the 18-line window condition, coupled with the similarity in proportions of discourse functions fitting the discourse plan schema, argues against the notion that message size per se moderates compression. Compression thus appears to be robust in synchronous CMC. In particular, similar amounts of decision making apparently occurred across experiments that differed in window size, task complexity, time available to complete the task (people in the earlier study planned two tasks rather than one), and other changes, such as message status information and how follow-up broadcasts were handled.

At the same time, we verified that our subjects generally preferred small messages, as the average size of a transmission involved 1.1 lines in the 4-line condition, and 1.7 lines in the 18-line condition. Hence, despite the same level of adherence to the discourse schema, there were some differences because of the manipulated software features. Indeed, it is likely that different characteristics of CMC can affect message size. In Colomb and Simutis (1996), for example, extraordinarily short transmissions were found that appeared to be due to interactions involving a number of people being on line simultaneously. The rapid scrolling of messages from multiple users of the system would exert strong pressure for short messages, because of the likelihood of a topic or thread being deactivated by the time a long message is composed and sent.

The differences we found that were due to text window size went beyond size of the message, however, as they suggested the emergence of different communicative and discourse management strategies in the various conditions. Specifically, people in the 10-line condition apparently had greater (and, as it proved, unnecessary) difficulty in meeting the constraints of composing a large message within the allowed window, as is evidenced by the greater proportion of backspaced material. In addition, long turn strategies (a cooperative strategy of making a number of decisions within a single turn), resulting in high-density discourses, were more common in the 18-line condition, but the 4-line condition exhibited a greater difference in the relative contributions of the partners to the discourse.

Thus, our results contain both a caution and a promise. The caution is that differences in face-to-face communication and CMC may be due to the specifics of the CMC interface being used, as people develop emergent strategies in response to the specific design decisions of a given program. Sometimes striking differences may be found (as is the case when people do the MTV problem over e-mail; see Condon \& Cech, 1998). In contrast, the promise is that the discovery of discourse characteristics that are robust over a particular category of CMC potentially enables the use of new and powerful techniques for the study of theories of conversation.

We believe that the need for such techniques arises from the importance of studying discourse in an environment that involves the actual construction or production of a conversation rather than the passive reception of written or spoken discourse. This follows from one of the major conclusions emerging from work in conversational analysis: Conversational discourses involve the joint construction of a discourse structure (see, e.g., Sacks, Schegloff, \& Jefferson, 1974; Schegloff, 1982; see also van Dijk, 1997a, 1997b) rather than autonomous contributions. This argument increasingly is being accepted in other disciplines, such as psychology (see, e.g., Clark, 1997; Clark \& Schaefer, 1989; Clark \& Wilkes-Gibbs, 1986; Schober \& Clark, 1989) and linguistics (see recent work on speech act theory by Geis, 1995). The work of Clark and his colleagues and students (see, e.g., Clark, 1994) has demonstrated some ways in which experimental techniques may be applied to the study of authentic conversation.

Another method that may prove useful for studying ongoing conversation involves the construction of software to intervene in and alter messages in synchronous CMC. In principle, such intervention techniques may enable strong tests of psycholinguistic theories, in a manner analogous to that in which intervention techniques in the eye-tracking paradigm are used to study reading (see, e.g., McConkie \& Rayner, 1975; Rayner, 1975). Intervention techniques that rely on computer technology have led to significant advances, not only in reading (e.g., see also Aaronson \& Colet, 1997; Rayner \& Pollatsek, 1989), but also, and more recently, in the study of writing and composition (see Gettys \& Berglau, 1989; Levy, 1997; Levy \& Ransdell, 1994; Ransdell, 1990). As we learn more about emergent and robust discourse strategies across different media and different communicative interfaces within a medium, we open up the possibility of similar advances through controlled experiments utilizing CMC. 


\section{REFERENCES}

Aaronson, D., \& Colet, E. (1997). Reading paradigms: From lab to cyberspace? Behavior Research Methods, Instruments, \& Computers, 29, 250-255.

ANderson, J. F., Beard, F. K., \& Walther, J. B. (1998). The local management of computer-mediated conversation. Manuscript submitted for publication.

BAYM, N. K. (1996). Agreements and disagreements in a computermediated discussion. Research on Language \& Social Interaction, 29, 315-345

BordiA, P. (1997). Face-to-face versus computer-mediated communication: A synthesis of the experimental literature. Journal of Business Communication, 34, 99-120.

ClaRk, H. H. (1994). Discourse in production. In M. A. Gernsbacher (Ed.), Handbook of psycholinguistics (pp. 985-1021). San Diego Academic Press.

ClaRK, H. H. (1996). Using language. Cambridge: Cambridge University Press.

ClaRK, H. H. (1997). Dogmas of understanding. Discourse Processes, $23,567-598$

Clark, H. H., \& Schaefer, E. F. (1989). Contributing to discourse Cognitive Science, 13, 259-294.

Clark, H. H., \& Wilkes-GibBS, D. (1986). Referring as a collaborative process. Cognition, 22, 1-39.

Colomb, G. G., \& Simutis, J. A. (1996). Visible conversation and academic inquiry: CMC in a culturally diverse classroom. In S. Herring (Ed.), Computer-mediated communication: Linguistic, social, and cross-cultural perspectives (pp. 203-222). Philadelphia: Benjamins.

Condon, S. L., \& CECH, C. G. (1992). Manual fur coding decisionmaking interactions. Unpublished manuscript.

CONDON, S. L., \& ČECH, C. G. (1996a). Discourse management strategies in face-to-face and computer-mediated decision-making interactions. Electronic Journal of Communication /La Revue Electronique de Communication, 6 (3).

CONDON, S. L., \& C to-face and computer-mediated decision-making interactions. In S. Herring (Ed.), Computer-mediated communication: Linguistic, social, and cross-cultural perspectives (pp. 65-80). Philadelphia: Benjamins

Condon, S. L., \& CECH, C. G. (1998). Computer mediated discourse management in three modalities. Manuscript submitted for publication

Condon, S., Cooper, C., \& Grotevant, H. (1984). Manual for the analysis of family discourse. Psychological Documents 14.1, Document no. 2616

Ess, C. (ED.) (1996). Philosophical perspectives on computer-mediated communication. Albany: State University of New York Press.

FERRERIA, F., \& ANES, M. (1994). Why study spoken language? In M. A. Gernsbacher (Ed.), Handbook of psycholinguistics (pp. 33-56). San Diego: Academic Press.

GeIs, M. (1995). Speech acts and conversational interaction. Cambridge: Cambridge University Press.

GeTTYS, C. F., \& BERGLAU, L. (1989). Laboratory MicroStar: A datalogging word processor for research on word processing. Behavior Research Methods, Instruments, \& Computers, 21, 484-486.

HERRING, S. (ED.) (1996). Computer-mediated communication: Linguistic, social, and cross-cultural perspectives. Philadelphia: Benjamins.

Hiltz, S. R., Johnson, K., \& Turoff, M. (1986). Experiments in group decision-making: Communication process and outcome in face-to-face versus computerized conferences. Human Communication Research, $13,225-252$

Kiesler, S., Zubrow, D., Moses, A., \& Geller, V. (1985). Affect in computer-mediated communication: An experiment in synchronous terminal-to-terminal discussion. Human-Computer Interaction, 1, 77-104

Ko, K. (1996). Structural characteristics of computer-mediated language: A comparative analysis of InterChange discourse. Electronic Journal of Communication/La Revue Electronique de Communication, 6 (3).
Lea, M., O'Shea, T., Fung, P., \& Spears, R. (1992). "Flaming" in computer-mediated communication: Observations, explanations, implications. In M. Lea (Ed.), Contexts of computer-mediated communication (pp. 89-112). New York: Harvester Wheatsheaf.

Levinson, B. (1983). Pragmatics. Cambridge: Cambridge University Press.

LEVY, C. M. (1997). The "R" that psychology forgot: Research on writing processes. Behavior Research Methods, Instruments, \& Computers, 29, 135-145.

LEVY, C. M., \& RANSDELL, S. (1994). Computer-aided protocol analysis of writing processes. Behavior Research Methods, Instruments, \& Computers, 26, 219-223.

Li, J., Seu, J. H., Evens, M., Michael, J. A., \& Rovick, A. A. (1992). Computer dialogue system (CDS): A system for capturing computermediated dialogue. Behavior Research Methods, Instruments, \& Computers, 24, 535-540.

MCCONKIE, G. W., \& RAYNeR, K. (1975). The span of the effective stimulus during a fixation in reading. Perception \& Psychophysics, 17, 578-586.

RANSDELL, S. E. (1990). Using a real-time replay of students' word processing to understand and promote better writing. Behavior Research Methods, Instruments, \& Computers, 22, 142-144.

RAYNER, K. (1975). The perceptual span and peripheral cues in reading. Cognitive Psychology, 7, 65-81.

RAYNER, K., \& PollatSEK, A. (1989). The psychology of reading. Englewood Cliffs, NJ: Prentice-Hall.

SACKS, H. (1973). Lecture notes. Ann Arbor: University of Michigan, Summer Institute of Linguistics.

SACKS, H., Schegloff, E., \& JefFerson, G. (1974). A simplest systematics for the organization of turn-taking for conversation. Language, 50, 696-735.

SCHEGLOFF, E. (1968). Sequencing in conversational openings. American Anthropologist, 70, 1075-1095.

SCHEGLOFF, E. (1982). Discourse as an interactional achievement: Some uses of "uh huh" and other things that come between sentences. In D. Tannen (Ed.), Analyzing discourse: Text and talk (pp. 71-93). Washington, DC: Georgetown University Press.

SCHEgloff, E., \& SACKs, H. (1973). Opening up closings. Semiotica, 8, 289-327.

SCHOBER, M. F., \& ClaRK, H. H. (1989). Understanding by addresses and overhearers. Cognitive Psychology, 21, 211-232.

Seu, J., Chang, R., Li, J., Evens, M., Michael, J., \& Rovick, A. (1991). Language differences in face-to-face and keyboard-tokeyboard tutoring sessions. Proceedings of the Cognitive Science Conference (pp. 576-580). Hillsdale, NJ: Erlbaum.

Siegel, J., Dubrovsky, V., Kiesler, S., \& McGuire, T. (1986). Group processes in computer-mediated communication. Organizational Behavior \& Human Decision Processes, 37, 157-186.

SPEARS, R., \& LEA, M. (1994). Panacea or panopticon? The hidden power in computer-mediated communication. Communication Research, 21, $427-459$

SProull, L., \& KieSLer, S. (1991). Connections: New ways of working in the networked organization. Cambridge, MA: MIT Press.

TANNEN, D. (ED.) (1982). Spoken and written language: Exploring orality and literacy. Norwood, $\mathrm{NJ}$ : Ablex.

VAN DıJK, T. A. (ED.) (1997a). Discourse as social interaction. London: Sage.

VAN DIJK, T. A. (ED.) (1997b). Discourse as structure and process. London: Sage.

WEISBAND, S. P. (1992). Group discussion and first advocacy effects in computer-mediated and face-to-face decision making groups. Organizational Behavior \& Human Decision Processes, 53, 352-380.

(Manuscript received November 4, 1997; revision accepted for publication February 9, 1998.) 